\title{
Intensive Home Blood Pressure Lowering in Advanced Chronic Kidney Disease: A Pilot Randomized Controlled Trial Protocol
}

\author{
Se Ri Bae' \\ Charles E McCulloch ${ }^{2}$ \\ Raymond $\mathrm{K} \mathrm{Hsu}$ (iD),* \\ Elaine $\mathrm{Ku}^{\mathrm{l}, 3, *}$ \\ 'University of California, San Francisco, \\ Division of Nephrology, Department of \\ Medicine, San Francisco, CA, USA; \\ ${ }^{2}$ University of California, San Francisco, \\ Department of Epidemiology and \\ Biostatistics, San Francisco, CA, USA; \\ ${ }^{3}$ University of California, San Francisco, \\ Division of Pediatric Nephrology, \\ Department of Pediatrics, San Francisco, \\ CA, USA \\ *These authors contributed equally to \\ this work
}

Background: Suboptimal blood pressure (BP) control is known to be common in patients with advanced chronic kidney disease (CKD) and may contribute to elevated risk of cardiovascular $(\mathrm{CV})$ morbidity and mortality. Most trials have tested alternative clinicbased BP targets, even though home and ambulatory BPs have been shown to be more strongly associated with outcomes of interest. We designed a pilot randomized controlled trial to examine whether intensive lowering of systolic blood pressure (SBP) to a target $<120$ $\mathrm{mmHg}$ with remote home BP monitoring is safe and feasible for patients with advanced $\mathrm{CKD}$ as they transition to end-stage kidney disease (ESKD).

Methods: Patients with stage 4 or 5 CKD (estimated glomerular filtration rate $\leq 30 \mathrm{~mL} / \mathrm{min}$ / $1.73 \mathrm{~m}^{2}$ ) with hypertension, defined as receipt of at least one antihypertensive agent or a clinic SBP $>140 \mathrm{mmHg}$, will be randomly assigned to either a home SBP target of $<120$ $\mathrm{mmHg}$ (intervention group) or $130-140 \mathrm{mmHg}$ (control group). Antihypertensive medications are titrated as needed to achieve target SBP levels over the first 4 months of the trial, and target SBPs are maintained thereafter. After month 12, biannual visits are conducted until study closeout. Participants are followed closely for safety and feasibility outcomes including screening:enrollment ratio, rates of hyperkalemia, hospitalizations or emergency room visits, and achieved separation in home BP readings between the two groups at month 8 .

Discussion: This protocol outlines the design of a pilot trial to examine the safety and feasibility of an intensive home BP lowering intervention in patients with advanced CKD through remote home BP monitoring. This pilot study will provide the necessary data to inform the planning of a full-scale trial comparing strict versus usual BP control in patients with advanced CKD, including those with diabetes. Knowledge of the optimal BP targets in advanced CKD will better inform the approach to CV and kidney risk reduction as patients transition to ESKD.

Trial Registration: ClinicalTrials.gov identifier NCT02975505. Registered November 29, 2016. https://clinicaltrials.gov/ct2/show/NCT02975505?term=NCT02975505\&draw=2\&rank=1.

Keywords: ESKD, CKD, hypertension

\section{Introduction}

Hypertension, a major modifiable cardiovascular risk factor, is present in over $90 \%$ of patients with stage 4 or $5 \mathrm{CKD}$, and the percentage of patients with $\mathrm{BP}<140 / 90 \mathrm{mmHg}$ is reportedly as low as $13 \%$ in some studies. ${ }^{1-3}$ Given that many patients who reach CKD stage 4 will survive to develop end-stage kidney disease [ESKD], ${ }^{4-6}$ and that cardiovascular $(\mathrm{CV})$ events are the leading cause of death in ESKD, ${ }^{4,7}$ intensive BP control could reduce the high morbidity, mortality, and costs associated with ESKD care.
Correspondence: Elaine $\mathrm{Ku}$ Division of Nephrology, Department of Medicine, University of California, San Francisco, 533 Parnassus Avenue, U404 Box 0532, San Francisco, CA, 94I43-0532, USA

Tel + | 4I5-476-7696

Fax + | 415-476-9976

Email elaine.ku@ucsf.edu 
Prior BP trials in patients with moderate CKD (mostly stage 3) did not demonstrate a kidney benefit to the intensive lowering of SBP to a $<140 / 90 \mathrm{mmHg}$ target, but the recent Systolic Blood Pressure Intervention Trial (SPRINT) demonstrated that lowering SBP to $<120$ $\mathrm{mmHg}$ reduced the risk of $\mathrm{CV}$ events and mortality in patients over the age of 50 with high $\mathrm{CV}$ risk and baseline SBP of $130-180 \mathrm{mmHg}{ }^{8}$ However, in SPRINT, participants with eGFR $<20 \mathrm{~mL} / \mathrm{min} / 1.73 \mathrm{~m}^{2}$ and difficult-tocontrol hypertension were excluded, and only a few patients with eGFR $<30 \mathrm{~mL} / \mathrm{min} / 1.73 \mathrm{~m}^{2}$ were enrolled. Because SPRINT was terminated early, the duration of follow-up may not have been long enough to detect differences in kidney outcomes. Of note, the African American Study of Kidney Disease and Hypertension (AASK) trial showed that intensive BP lowering (mean arterial pressure [MAP] $\leq 92 \mathrm{mmHg}$ ) in African Americans with hypertensive kidney disease did not slow the progression of kidney disease when compared to less intensive BP control (MAP 102-107). ${ }^{9}$ However, like SPRINT, patients with eGFR $<20 \mathrm{~mL} / \mathrm{min} / 1.73 \mathrm{~m}^{2}$ were excluded from this study.

One of the few trials that tested intensive BP control in patient with advanced CKD was the Modification of Diet in Renal Disease (MDRD) Trial, which randomized patients with glomerular filtration rate between 13 and $24 \mathrm{~mL} / \mathrm{min} / 1.73 \mathrm{~m}^{2}$ to MAP of $\leq 92 \mathrm{mmHg}$ versus $\leq 107$ mmHg. ${ }^{10}$ Subgroup analysis of the patients with advanced CKD did not show a kidney benefit to intensive BP control during the parent trial (which had a mean duration of only two years of follow-up), although during post-hoc longterm follow-up there appeared to be an all-cause ESKD benefit to strict BP control. ${ }^{11}$ A study combining MDRD and AASK participants also showed that strict BP control reduced the risk of death among patients with eGFR $<30 \mathrm{~mL} / \mathrm{min} / 1.73 \mathrm{~m}^{2}$ and slowed the progression to ESKD among those with higher levels of proteinuria. These findings indicate that intensive BP lowering may be particularly beneficial for these subgroups of patients with advanced CKD. ${ }^{12,13}$

In current clinical practice, a large proportion of patients with advanced CKD do not achieve lower BP targets (eg, $\mathrm{SBP}<120 \mathrm{mmHg}$ ), which is likely due to a variety of factors. ${ }^{2,14-16}$ First, intensive BP control would require the use of additional antihypertensive agents. Providers often avoid use of renin-angiotensin-aldosterone system (RAAS) inhibitors in patients with severe kidney dysfunction due to concerns regarding the risk for hyperkalemia, even though RAAS inhibitors are likely to be effective antihypertensive agents for this population. ${ }^{17}$ Second, the pill burden during the advanced stages of CKD is already high and intensifying BP control would require the use of additional medications, which patients may be unwilling to comply with. ${ }^{18,19}$ Third, some studies have suggested that tight BP control during the advanced stages of CKD may predispose patients to acute kidney injury (AKI), ${ }^{20}$ which may in turn increase the risk of ESKD. Fourth, few trials have included a substantial number of patients with advanced CKD, so data on the benefits of intensive BP control are mostly extrapolated from patients with mild or moderate severity of CKD.

A major limitation in the current literature is the lack of evidence surrounding optimal BP targets in patients with diabetes ${ }^{21}$ as most large trials have primarily enrolled patients with CKD and without diabetes. ${ }^{10,22}$ It remains unclear whether the benefits of intensive BP control would be similar in those with diabetes (compared to those without diabetes), although some post-hoc analyses have suggested similar cardiovascular benefits. ${ }^{23}$ Evidence that suggests that the advanced CKD population, including those with diabetes, can be safely recruited and retained in clinical trials of intensive BP control may better inform the clinical management of a large proportion of the population with advanced CKD and potentially improve adherence to BP guideline recommendations.

Most BP trials in adults have tested different clinic BP targets, even though home and ambulatory BPs have been shown to be more strongly associated with adverse kidney and cardiovascular outcomes than clinic BP measurements. ${ }^{24-26}$ Home BP readings have also been shown to reduce misclassification of white-coat and masked hypertension in patients with $\mathrm{CKD} .^{27}$ Data on whether targeting home BPs, as opposed to clinic BPs, is feasible are needed in the advanced CKD population, as this strategy can reduce the number of provider visits needed to achieve adequate BP control and enhance patient awareness of their own BP levels. ${ }^{28-30}$

Given the potential benefits of strict BP control (SBP target $<120 \mathrm{mmHg}$ ) along with the uncertainty of the optimal BP targets in the advanced CKD population, the current protocol describes the design for a pilot trial that examines the safety and feasibility of randomly assigning patients with advanced CKD to a home SBP $<120 \mathrm{mmHg}$ (intervention group) or a home SBP target of 130-140 $\mathrm{mmHg}$ (usual care group) using home BP measurements. We hypothesize that among patients with or without a history of diabetes, achievement of intensive BP lowering ( $\mathrm{SBP}<120 \mathrm{mmHg}$ ) by home $\mathrm{BP}$ measurements in the 
setting of low kidney function (eGFR $\leq 30 \mathrm{~mL} / \mathrm{min} /$ $1.73 \mathrm{~m}^{2}$ ) can be achieved over an 8-month period.

\section{Methods}

\section{Study Design}

This pilot study is a non-blinded randomized controlled trial investigating the safety and feasibility of strict blood pressure control in patients with advanced CKD. Patients will be randomized in 2:1 ratio to intervention (home SBP target $<120 \mathrm{mmHg}$ ) or usual care (home SBP target 130-140 $\mathrm{mmHg}$ ) until 75 patients are enrolled, and then subsequently patients will be randomized in 1:1 ratio to intervention or usual care. Antihypertensive medications will be titrated as needed to achieve target home SBPs over the course of 4 months and maintained thereafter until study closeout (see Figure 1). Biannual visits are conducted after month 12 .

\section{Study Population}

All patients are recruited from the University of California San Francisco nephrology clinics. Inclusion and exclusion criteria are as follows:

\section{Inclusion Criteria}

1. $\geq 18$ years of age.

2. English-speaking.

3. Patients with advanced CKD who meet the eGFR criteria defined as either:

a. At least two eGFRs $\leq 30 \mathrm{~mL} / \mathrm{min} / 1.73 \mathrm{~m}^{2}$ in the last three months.

b. A prior diagnosis of CKD and at least one eGFR $\leq 30 \mathrm{~mL} / \mathrm{min} / 1.73 \mathrm{~m}^{2}$ in the last three months.
4. Presence of elevated BP, defined as receipt of one or more antihypertensive agents or an office SBP $>140$ $\mathrm{mmHg}$ at the time of the screening visit. If the participant is not on antihypertensive medications, they must have an average home $\mathrm{SBP}>130 \mathrm{mmHg}$ during the first week of the study when baseline home BPs are ascertained.

5. Participants must have a mid-arm circumference between 22 and $37 \mathrm{~cm}$ and access to a smart phone or tablet in order to use the Bluetoothenabled home BP device, QardioArm ${ }^{\odot}$.

\section{Exclusion Criteria}

Patients are excluded if they:

1. Are planning to become pregnant or are currently pregnant.

2. Are marginally housed due to concerns regarding follow-up.

3. Are institutionalized or a prisoner.

4. Are actively participating in a different interventional trial that may affect blood pressure.

5. Are actively abusing illicit drugs and alcohol.

6. Have a history of poor compliance.

7. Take $>5$ antihypertensive medications (including diuretics) at maximum doses.

8. Have cognitive impairment and are unable to follow instructions regarding home BP measurement during screening visit.

If patients meet all inclusions and exclusion criteria, we will check with their nephrology providers prior to

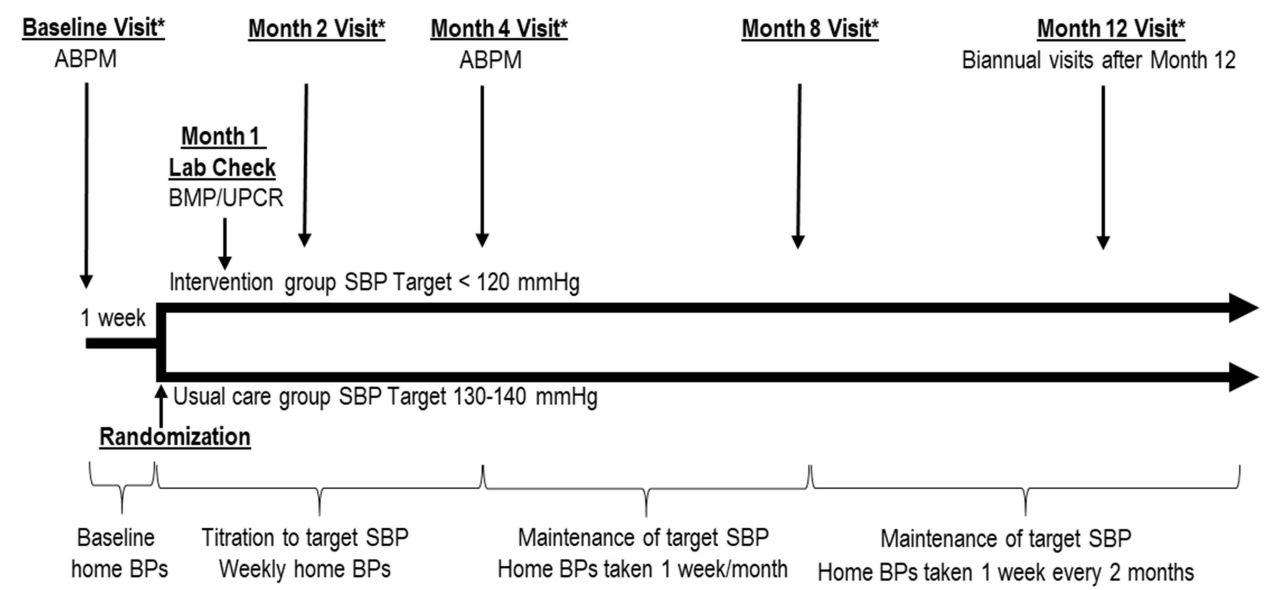

Figure I Study Design and Timeline. *Indicates an in-person visit, which includes BMP/UPCR, physical exam, and office BPs. Abbreviations: BMP, basic metabolic panel; UPCR, urine protein creatinine ratio; ABPM, ambulatory blood pressure monitoring. 
approaching participants to ensure their candidacy in our study.

\section{Baseline Visit}

During the baseline visit, participant eligibility is confirmed, written consent is obtained, and demographic, co-morbidity, and laboratory data are collected. A brief physical examination is performed and three automated office BP readings in the seated position followed by two standing BP readings are recorded one minute apart after five minutes of quiet rest. At the baseline visit, enrollees are provided with a QardioArm ${ }^{\mathcal{O}}$ device and trained to use the device to take standardized home BP measurements.

Participants are asked to take home BPs for a one-week baseline period to ensure continued eligibility and compliance with our protocol prior to randomization, with a minimum of 6 readings required over the one-week period. Participants are also asked to complete 24-hour ambulatory blood pressure monitoring (ABPM) during their baseline period. Patients who refuse to undergo 24hour ABPM are not excluded from study.

\section{Randomization}

Eligible patients who complete the one week of baseline home readings will be randomized to either a home SBP target of $<120 \mathrm{mmHg}$ (intervention group) or 130-140 mmHg (usual care group) in 2:1 ratio until 75 participants are enrolled, and participants will be subsequently randomized in 1:1 ratio thereafter. The rationale for the 2:1 ratio for the first 75 patients is to ensure that we initially capture enough safety data on participants randomized to intervention, but we plan to transition to $1: 1$ ratio randomization thereafter. We will conduct a sensitivity analysis comparing the effect during the 2:1 randomization phase of the trial and 1:1 phase to determine if there is an interaction between the intervention and the phase of the trial.

Once patients are screened and deemed eligible, study personnel will notify nephrologists of their patients' eligibility and obtain assent for patient participation in the trial prior to randomization. Stratified permuted-block randomization will be performed with stratification by diabetes status in order to ensure that patients with diabetes who have previously been excluded from many trials of intensive BP lowering ${ }^{8-10}$ are represented in our pilot study. Investigators will be blinded to the ordering of the randomization blocks and their respective sizes. The generation of allocation sequence is performed by and only accessible to the study coordinator for assignment of participants to the intervention and not accessible to investigators. Patients are contacted via phone or secure messaging to begin active titration of their antihypertensive regimens to achieve their assigned target SBPs after randomization.

\section{Home Blood Pressure Monitor}

QardioArm $^{\odot}$ is a wireless Bluetooth-enabled home BP monitor that is validated by the European Society of Hypertension Protocol and the American Heart Association $^{31}$ and has a strong direct linear correlation $(r=0.96)$ with the previously validated and widely accepted Omron $\mathrm{M} 3{ }^{\odot}$ BP device. ${ }^{32}$ The device is compatible with most smartphones and allows for real-time wireless transmission of BP readings through an online phone application. The device is also accompanied by a secure platform used by providers for BP data transmission, monitoring, download, and storage. All participants are provided a device at no cost, and Bluetooth linkage between the device and smartphone is setup by study personnel at the baseline visit.

\section{Office BP Readings During Study Visits}

Office BPs are measured at the baseline, 2, 4, 8, and 12month study visits (Figure 1), and every six months thereafter. Participants are seated quietly in a room with the study coordinator for 5 minutes before BPs are taken. Three measurements are taken one minute apart. Participants are then asked to stand for two minutes and two standing BPs are recorded one minute apart. One study coordinator will be present during all office BP measurements.

\section{Intervention}

\section{Blood Pressure Targets}

We have chosen to target home SBP, as opposed to MAP or diastolic BP (DBP) based primarily on the CV and mortality benefits seen recently in SPRINT $^{8}$ and data that suggest that SBP is a stronger predictor of ESKD risk and CV disease than DBP or MAP. ${ }^{24,33,34}$ Our usual care group will be treated to home SBP targets of $130-140 \mathrm{mmHg}$ (equivalent to office SBP of $135-145 \mathrm{mmHg}$ ) because this was the range of BPs that screened patients had at baseline enrollment under routine care. During routine clinical care, any non-study provider can titrate antihypertensive agents as per their preference outside of the study protocol based on the clinic BP readings and other data available to them, and such titrations are noted and will be accepted by the study team. Home BP readings are made readily available to non-study providers upon request, and all nephrology 
clinic providers are alerted of patient enrollment in the study and the assigned BP target following randomization.

\section{Home Blood Pressure Readings}

Participants are asked to measure their BPs at home on any three days of the week. For each day, participants take 3 readings in the morning and 3 in the evening. Participants are instructed to rest for 5 minutes before taking readings and sit quietly for 1 minute in between readings. Weekly home BP averages are calculated from the most recent 18 readings in a given week. Antihypertensive medications will be titrated by study investigators based on the average of the most recent 18 home BP measurements received, with a minimum of 6 required readings per week to be considered sufficient for any changes to medications. An insufficient number of measurements will prompt contact with the participant to increase the number of readings.

\section{Antihypertensive Therapy Titration Intervention}

Antihypertensive medications are titrated during the first 4 months to achieve target home SBP levels in both the intervention and usual care groups if home BP readings are not already within the target range. The antihypertensive therapy titration intervention will be individualized according to each patient's medical history, current medication regimen, and medication preference as specified by their nephrologist. Participants' referring nephrologist will be informed of their participation at the time of enrollment and asked to indicate which BP agents they would prefer to withdraw or add if SBP is outside of target according to their own practice patterns. Doses of current antihypertensive agents may be preferentially increased or decreased first depending on patient's home BP readings relative to their target SBP. This will be the first-line approach to avoid introduction of multiple new medications if doses of current medications are not yet optimized. New agents may be prescribed or discontinued after careful consideration of patient's medical history and nephrologist's input. Combination drugs will be acceptable but only used if suggested by the participant's nephrologist, as there may be cost barriers to the use of combination drugs. Although every antihypertensive treatment regimen will likely differ for each participant, the suggested order of antihypertensive agent titration (if the nephrologist does not indicate a specific preference) will be angiotensin receptor blockers (ARBs) or angiotensin-converting enzyme inhibitors (ACEi) as first-line therapy, followed by (in order of preference) diuretics, calcium-channel blockers, betablockers, vasodilators/nitrates, sympatholytics, alphablockers, and potassium-sparing diuretics. See Figure 2 for the antihypertensive medication titration protocol.

We will permit up to a $30 \%$ increase in serum creatinine level after ACEi/ARB initiation or uptitration of dosages of other antihypertensive medications, in accordance with traditional guidelines and published literature. ${ }^{35,36}$ For patients who experience $>30 \%$ increase in serum creatinine, the ACEi/ARB dose or other antihypertensive medication will be reduced, with plans for a repeat serum creatinine within 2 weeks. If the serum creatinine continues to be elevated, then the ACEi/ARB will be discontinued. We will also respect the opinion of the patient's nephrologist on this issue. If a nephrologist is concerned about any rise in serum creatinine, we will reduce or discontinue the drug. We will then proceed with the next suggested class of antihypertensive agents to help achieve the target blood pressure.

During the first 8 months of the study, participants will be contacted every other week by study coordinators to note any adverse events including dizziness, falls, syncope, hospitalizations, emergency room visits, acute kidney injury, CV events, or allergic reactions, as well as adherence to medication regimen and medication side effects such as diuresis, impotence, cough, lack of energy and sleepiness, lightheadedness on standing, or headaches.

\section{Laboratory Tests}

Basic metabolic panel and urine chemistry laboratory tests are obtained at baseline and at months 1, 2, 4, 8, 12, and every six months thereafter but timed as closely to clinical laboratory tests as possible (to avoid additional burden on participants) in a pragmatic approach. Labs are closely monitored by study investigators and medications are titrated accordingly to ensure the safety of all participants. Additional laboratory testing is performed as needed.

\section{Follow-Up Timeline}

The timeline of study visits during the one-year of followup is outlined in Figure 1. Participants are instructed to take weekly home BP readings for the first 4 months of the study, or until target SBP levels are achieved for two consecutive weeks. Once participants reach target SBP levels, home BP readings can be taken on a monthly basis over a one-week period each month. If participants maintain their target SBP levels after 8 months, home BP readings can be taken every two months thereafter. Participants will maintain their respective SBP target 


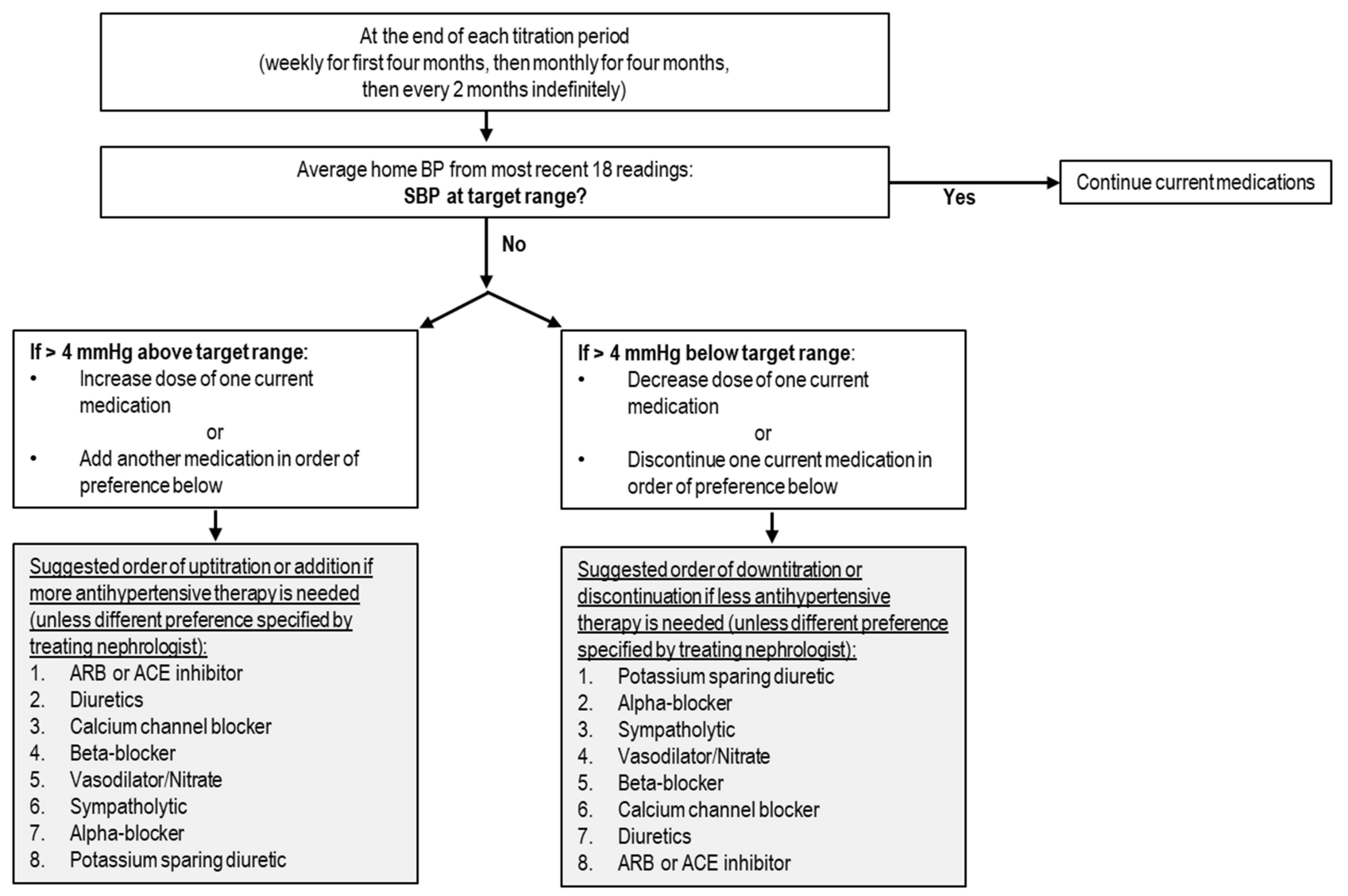

Figure 2 Antihypertensive medication titration protocol.

Abbreviations: BP, blood pressure; SBP, systolic blood pressure; ARB, angiotensin receptor blocker; ACE, angiotensin-converting enzyme.

levels for the remainder of the study after month 8 . If mean weekly SBP readings are $>4 \mathrm{mmHg}$ outside the target range, antihypertensive therapy titration is resumed weekly until target SBP levels are achieved again for two consecutive weeks.

Participants are seen in person at Months 2, 4, 8, and 12 , and every 6 months thereafter, but visits will be timed as close to their clinical visits as possible to avoid additional burden on participants in a pragmatic approach. Inperson follow-up visits consist of a brief physical exam, office BP readings which will be obtained and used to calibrate the QardioArm ${ }^{\mathcal{C}}$ device to ensure accuracy of its readings, and laboratory testing. Patients will be contacted throughout the study via phone or secure text messages depending on patient preference. Adherence to antihypertensive therapy treatment will be assessed by administering a questionnaire during every in-person visit and follow-up phone call to ask participants if they are taking antihypertensive medications as prescribed and if they missed any doses since last follow-up. Adverse events (including emergency room visits or hospitalizations) and onset of ESKD, defined as initiation of chronic dialysis or receipt of kidney transplant, are also ascertained during follow-up. Participants will be followed indefinitely unless they choose to disenroll from the study or are lost to follow-up.

\section{Outcomes Measures}

The primary outcome will be feasibility of achieving at least a $6 \mathrm{mmHg}$ separation between intensive BP control and usual BP control arms at month 8 . A secondary outcome will be the difference in BP between the two treatment strategies at study closeout. Adherence to home BP monitoring will be measured by the number of weeks participants successfully complete the minimum number of home readings.

Safety of our intervention will also be a secondary outcome and will be determined by a composite of adverse events including hyperkalemia ( $\mathrm{K} \geq 6.0 \mathrm{meq} / \mathrm{L})$, selfreported falls, syncope, or acute kidney injury (AKI) 
requiring emergency room visit or hospitalizations. Discharge summaries from emergency room visits and hospitalizations in patient health records will be reviewed and events adjudicated by two study investigators. In addition, onset of ESKD will not be a censoring event; patients will be encouraged to continue to check home BPs following transplant or dialysis, but no study-driven active titration of antihypertensive agents will occur after ESKD onset.

\section{Statistical Analysis}

Safety outcomes, or rates of adverse events, will be tallied using simple proportions and descriptive statistics. Intention-to-treat analysis will be performed. Stratified analyses by diabetes status will be performed given the lack of robust trial data to support intensive BP lowering in patients with diabetes. We will also test for differences in the composite rates of hospitalizations, emergency room visits, and hyperkalemia in the two arms. We will explore differences in the rates of ESKD or cardiovascular events and determine if there is any effect modification of the intervention by level of proteinuria or by diabetes. For patients lost to follow-up, we plan to use chained multiple imputation to perform analyses.

\section{Sample Size Rationale}

Randomizing 96 participants to strict vs usual BP control will provide $80 \%$ power (with 2 -sided $\alpha$ of 0.05 ) to detect a 6-mmHg difference in SBP (with SD difference of 10 $\mathrm{mmHg}$ ) between the two arms at month 8. Anticipating approximately a $25 \%$ dropout rate, we plan to enroll 120 participants. An effect size of similar magnitude has been reported previously in tele-monitoring feedback from pharmacists in adult home BP monitoring trials. ${ }^{37}$

\section{Discussion}

This randomized controlled pilot trial aims to examine the safety and feasibility of an intensive BP lowering intervention in patients with advanced CKD through remote home BP monitoring. The innovation of this protocol includes the management of BPs using smartphone technology and targeting of home as opposed to clinic BPs. Additionally, patients with advanced (stage 4 or 5) CKD represent an understudied population who are frequently excluded from clinical trials. Demonstration that this population can be safely recruited, retained, and intervened upon will help promote their inclusion in future clinical trials.
Practical issues that we foresee in implementing the study include non-compliance among participants due to increased pill burden and concerns over changes in kidney function with intensification of BP control. Building strong rapport with participants will prove to be an essential component of ensuring compliance to study protocol. Participants who are unfamiliar with smartphone technology may also require additional training and support to obtain home BP measurements. Additionally, the pragmatic approach of scheduling in-person study follow-up dates to coincide with clinical visit appointments may also result in slight discrepancies between expected follow-up and actual study visit dates.

Despite these practical barriers, this pilot study will provide the necessary data to inform the planning of a fullscale trial that can help elucidate optimal BP targets in patients with advanced CKD and better inform management of $\mathrm{CV}$ and renal risk reduction as patients transition to ESKD.

\section{Abbreviations}

$\mathrm{BP}$, blood pressure; CKD, chronic kidney disease; $\mathrm{CV}$, cardiovascular; SBP, systolic blood pressure; ESKD, endstage kidney disease; SPRINT, Systolic Blood Pressure Intervention Trial; eGFR, estimated glomerular filtration rate; MDRD, Modification of Diet in Renal Disease; RAAS, renin-angiotensin-aldosterone system; AKI, acute kidney injury; BMP, basic metabolic panel; UPCR, urine protein creatinine ratio; ABPM, ambulatory blood pressure monitoring; DBP, diastolic blood pressure; MAP, mean arterial pressure; ARB, angiotensin receptor blockers; ACEi, angiotensin-converting enzyme inhibitor.

\section{Data Sharing Statement}

Data sharing is not applicable to this article as no datasets were generated or analyzed during the current study.

\section{Ethics Approval and Consent to Participate}

Ethics approval for the trial was obtained by the University of California, San Francisco Institutional Review Board (UCSF approval \#: 16-20978). Written informed consent from all participants will be obtained prior to enrollment. Data and safety monitoring for the trial will be the responsibility of an independent group of individuals who are not involved in the design, conception, or execution of our clinical trial and do not have 
proprietary interest in the outcome of the trial and will be performed when approximately half of participants have been enrolled. This trial will be conducted in accordance with the Declaration of Helsinki.

\section{Author Contributions}

All authors made substantial contributions to conception and design or analysis and interpretation of data; took part in drafting the article or revising it critically for important intellectual content; agreed to submit to the current journal; gave final approval of the version to be published; and agree to be accountable for all aspects of the work. SB is the study coordinator. EK, RH are the principal investigators. CEM is the biostatistician.

\section{Funding}

The trial is funded by the NIDDK K23 HL131023 to EK and UCSF Resource Allocation Program (RAP) grant to EK and RH. The authors have no financial relationships with Qardio or its affiliates.

\section{Disclosure}

Dr Charles E McCulloch and Dr Elaine $\mathrm{Ku}$ report grants from NIH, during the conduct of the study. The authors report no other conflicts of interest in this work.

\section{References}

1. Owen WF. Patterns of care for patients with chronic kidney disease in the United States: dying for improvement. J Am Soc Nephrol. 2004.

2. Sarafidis PA, Li S, Chen S-C, et al. Hypertension awareness, treatment, and control in chronic kidney disease. Am J Med. 2008;121 (4):332-340. doi:10.1016/j.amjmed.2007.11.025

3. Plantinga LC, Miller ER, Stevens LA, et al. Blood pressure control among persons without and with chronic kidney disease: US trends and risk factors 1999-2006. Hypertension. 2009;54(1):47-56. doi:10.1161/HYPERTENSIONAHA.109.129841

4. Saran R, Li Y, Robinson B, et al. US renal data system 2015 annual data report: epidemiology of kidney disease in the United States. $A m$ J Kidney Dis. 2016;67(3):A7-A8. doi:10.1053/j.ajkd.2015.12.014

5. Sud M, Tangri N, Levin A, Pintilie M, Levey AS, Naimark DM. CKD stage at nephrology referral and factors influencing the risks of ESRD and death. Am J Kidney Dis. 2014;63(6):928-936. doi:10.1053/j. ajkd.2013.12.008

6. O'Hare AM, Hotchkiss JR, Kurella Tamura M, et al. Interpreting treatment effects from clinical trials in the context of real-world risk information: end-stage renal disease prevention in older adults. JAMA Intern Med. 2014;174(3):391. doi:10.1001/jamainternm ed.2013.13328

7. Thompson S, James M, Wiebe N, et al. Cause of death in patients with reduced kidney function. $J$ Am Soc Nephrol. 2015;26(10):2504-2511. doi:10.1681/ASN.2014070714

8. Wright JT Jr, Williamson JD, Whelton PK, et al. A randomized trial of intensive versus standard blood-pressure control. $N$ Engl J Med. 2015;373(22):2103-2116. doi:10.1056/NEJMoa1511939
9. Wright JT, Bakris G, Greene T, et al. Effect of blood pressure lowering and antihypertensive drug class on progression of hypertensive kidney disease: results from the AASK trial. J Am Med Assoc. 2002. doi:10.1001/jama.288.19.2421

10. Klahr S, Levey AS, Beck GJ, et al. The effects of dietary protein restriction and blood-pressure control on the progression of chronic renal disease. $N$ Engl J Med. 2002.

11. Sarnak MJ, Greene T, Wang X, et al. The effect of a lower target blood pressure on the progression of kidney disease: long-term follow-up of the modification of diet in renal disease study. Ann Intern Med. 2005;142(5):342. doi:10.7326/0003-4819-1425-200503010-00009

12. Ku E, Sarnak MJ, Toto R, et al. Effect of blood pressure control on long-term risk of end-stage renal disease and death among subgroups of patients with chronic kidney disease. J Am Heart Assoc. 2019;8 (16):e012749. doi:10.1161/JAHA.119.012749

13. Sarafidis P, Loutradis C, Ortiz A, Ruilope LM. Blood pressure targets in patients with chronic kidney disease: MDRD and AASK now confirming SPRINT. Clin Kidney J. 2020;13(3).

14. Lash JP, Go AS, Appel LJ, et al. Chronic renal insufficiency cohort (CRIC) study: baseline characteristics and associations with kidney function. Clin J Am Soc Nephrol. 2009;4(8):1302-1311. doi:10.2215/ CJN.00070109

15. Cai G, Zheng Y, Sun X, Chen X. Prevalence, awareness, treatment, and control of hypertension in elderly adults with chronic kidney disease: results from the survey of prevalence, awareness, and treatment rates in chronic kidney disease patients with hypertension in China. J Am Geriatr Soc. 2013;61(12):2160-2167. doi:10.1111/ jgs. 12551

16. Muntner P, Anderson A, Charleston J, et al. Hypertension awareness, treatment, and control in adults with CKD: results From the Chronic Renal Insufficiency Cohort (CRIC) Study. Am J Kidney Dis. 2010;55 (3):441-451. doi:10.1053/j.ajkd.2009.09.014

17. Ku E, Lee BJ, Wei J, Weir MR. Hypertension in CKD: core curriculum 2019. Am J Kidney Dis. 2019;74(1):120-131. doi:10.1053/j. ajkd.2018.12.044

18. Becker GJ, Wheeler DC. Blood pressure control in CKD patients: why do we fail to implement the guidelines? Am J Kidney Dis. 2010;55(3):415-418. doi:10.1053/j.ajkd.2009.12.013

19. Muntner P, Judd SE, Krousel-Wood M, McClellan WM, Safford MM. Low medication adherence and hypertension control among adults with CKD: data from the REGARDS (Reasons for Geographic and Racial Differences in Stroke) Study. Am J Kidney Dis. 2010;56(3):447-457. doi:10.1053/j.ajkd.2010.02.348

20. Obi Y, Kalantar-Zadeh K, Shintani A, Kovesdy CP, Hamano T. Estimated glomerular filtration rate and the risk-benefit profile of intensive blood pressure control amongst nondiabetic patients: a post hoc analysis of a randomized clinical trial. J Intern Med. 2018;283(3):314-327. doi:10.1111/joim.12701

21. Cushman WC, Evans GW, Byington RP, et al.; ACCORD Study Group. Effects of intensive blood-pressure control in type 2 diabetes mellitus. N Engl J Med. 2010;362(17):1575-1585. doi:10.1056/ NEJMoa1001286

22. Appel LJ, Wright JT, Greene T, et al. Intensive blood-pressure control in hypertensive chronic kidney disease. $N$ Engl J Med. 2010;363 (10):918-929. doi:10.1056/NEJMoa0910975

23. Beddhu S, Chertow GM, Greene T, et al. Effects of intensive systolic blood pressure lowering on cardiovascular events and mortality in patients with type 2 diabetes mellitus on standard glycemic control and in those without diabetes mellitus: reconciling results from ACCORD BP and SPRINT. $J$ Am Heart Assoc. 2018;7(18). doi:10.1161/JAHA.118.009326

24. Agarwal R, Andersen MJ. Prognostic importance of ambulatory blood pressure recordings in patients with chronic kidney disease. Kidney Int. 2006;69(7):1175-1180. 
25. Agarwal R, Andersen MJ. Prognostic importance of clinic and home blood pressure recordings in patients with chronic kidney disease. Kidney Int. 2006;69(2):406-411. doi:10.1038/sj.ki.5000081

26. Agarwal R, Bills JE, Hecht TJW, Light RP. Role of home blood pressure monitoring in overcoming therapeutic inertia and improving hypertension control: a systematic review and meta-analysis. Hypertension. 2011;57 (1):29-38. doi:10.1161/HYPERTENSIONAHA.110.160911

27. Andersen MJ, Khawandi W, Agarwal R. Home blood pressure monitoring in CKD. Am J Kidney Dis. 2005;45(6):994-1001. doi:10.1053/j.ajkd.2005.02.015

28. Thilly N, Boini S, Kessler M, Briançon S, Frimat L. Management and control of hypertension and proteinuria in patients with advanced chronic kidney disease under nephrologist care or not: data from the AVENIR study (AVantagE de la Néphroprotection dans l'Insuffisance Rénale). Nephrol Dial Transplant. 2009;24 (3):934-939. doi:10.1093/ndt/gfn566

29. Patwardhan MB, Samsa GP, Matchar DB, Haley WE. Advanced chronic kidney disease practice patterns among nephrologists and non-nephrologists: a database analysis. Clin J Am Soc Nephrol. 2007;2(2):277-283. doi:10.2215/CJN.02600706

30. Nissenson AR, Collins AJ, Hurley J, Petersen H, Pereira BJ, Steinberg EP. Opportunities for improving the care of patients with chronic renal insufficiency: current practice patterns. J Am Soc Nephrol. 2001;12(8):1713-1720. doi:10.1681/ASN.V1281713

31. Chahine MN, Topouchian J, Zelveian P, et al. Validation of bp devices qardioarm $^{\circledR}$ in the general population and omron $m 6$ comfort $^{\circledR}$ in type ii diabetic patients according to the European Society of Hypertension international protocol (ESH-IP). Med Devices. 2018;11:11.
32. Mazoteras Pardo V, Losa Iglesias ME, López Chicharro J, Becerro de Bengoa Vallejo R. The QardioArm app in the assessment of blood pressure and heart rate: reliability and validity study. JMIR $m$ Health uHealth. 2017;5(12):e198-e198. doi:10.2196/mhealth.8458

33. Agarwal R. Blood pressure components and the risk for end-stage renal disease and death in chronic kidney disease. Clin J Am Soc Nephrol. 2009;4(4):830LP-837. doi:10.2215/CJN.06201208

34. Quiroga B, Galán I, García de Vinuesa S, Goicoechea M, Verdalles U, Luño J. Interarm systolic blood pressure as a predictor of cardiovascular events in patients with chronic kidney disease. Nephrol Dial Transplant. 2015;30(5):801-806. doi:10.1093/ndt/ gfv059

35. Bakris GL, Weir MR. Angiotensin-converting enzyme inhibitorassociated elevations in serum creatinine. Arch Intern Med. 2000;160(5). doi:10.1001/archinte.160.5.685

36. National Institute for Health and Care Excellence. Recommendations. Hypertension in adults: diagnosis and management. Guidance. NICE [Internet]. NICE Guideance. 2019:41. Available from: https://www. nice.org.uk/guidance/ng136/chapter/Recommendations\#startingantihypertensive-drug-treatment. Accessed June 4, 2021.

37. Uhlig K, Patel K, Ip S, Kitsios GD, Balk EM. Self-measured blood pressure monitoring in the management of hypertension. Ann Intern Med. 2013;159(3):185. doi:10.7326/0003-4819-159-3-20130806000008
Open Access Journal of Clinical Trials

\section{Publish your work in this journal}

The Open Access Journal of Clinical Trials is an international, peerreviewed, open access journal publishing original research, reports, editorials, reviews and commentaries on all aspects of clinical tria design, management, legal, ethical and regulatory issues, case record form design, data collection, quality assurance and data auditing

\section{Dovepress}

methodologies. The manuscript management system is completely online and includes a very quick and fair peer-review system, which is all easy to use. Visit http://www.dovepress.com/testimonials.php to read real quotes from published authors. 